\title{
Erratum: Interplay of magnetic order, pairing, and phase separation in a one-dimensional spin-fermion model [Phys. Rev. B 92, 115133 (2015)]
}

Wenjian Hu, Richard T. Scalettar, and Rajiv R. P. Singh

(Received 9 February 2016; published 7 March 2016)

DOI: 10.1103/PhysRevB.93.119903

We report a correction to the signs of two of the pairing terms in Eq. (6) in our paper. The correct version is

$$
M_{k}=\left[\begin{array}{cccc}
\epsilon_{k-q} & J & \Delta & 0 \\
J & \epsilon_{k} & 0 & -\Delta \\
\Delta & 0 & -\epsilon_{-(k-q)} & -J \\
0 & -\Delta & -J & -\epsilon_{-k}
\end{array}\right] .
$$

The corrected version of Figs. 7 and 8 are below. The central conclusion of our paper concerning phase separation at the spiral-antiferromagnetic (AF) transition in the neighborhood of $\mu=0$ (half-filling) remains valid. This phase separation was also reported previously in Ref. [1]. The primary difference from our previous results is that the lowest energy phase at low and high filling is $\mathrm{AF}$ rather than ferromagnetic $(\mathrm{F})$.

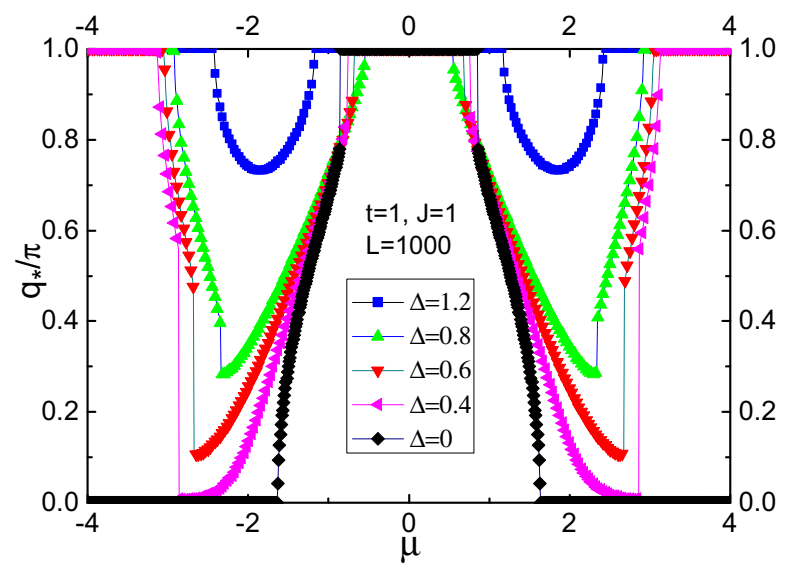

FIG. 7. Optimal wave vector $q_{*}$ that minimizes the ground-state energy $E_{0}$, as a function of chemical potential $\mu$ for fixed $J=1.0$ but different $\Delta$ values. Finite size effects are verified to be small.

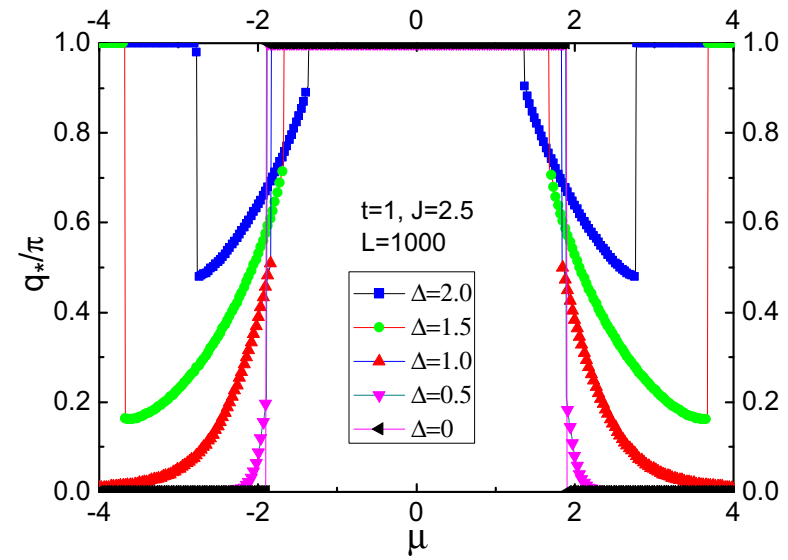

FIG. 8. Optimal wave vector $q_{*}$ that minimizes the ground-state energy $E_{0}$, as a function of chemical potential $\mu$ for fixed $J=2.5$ but different $\Delta$ values. 
The descriptions of the phase diagrams are now given by Figs. 9 and 10 below. The central features near $\mu=0$ are unchanged: $\mathrm{AF}$ in the vicinity of half-filling is replaced by spiral phases as $\mu$ deviates from $\mu=0$. Again, the changes are largely to the extremes of doping $(|\mu|>2)$ where AF replaces our previous report of F order. The underlying physical origin of the tendency to AF with $\Delta$ is that pairing favors equal numbers of up and down electrons, which is inconsistent with $\mathrm{F}$ ordering. This effect was insufficiently accounted for with the original signs of the pairing terms in Eq. (6). Additionally, $q=\pi$ magnetic order matches wave vector with the $q=\pi$ charge order, which tends to accompany pairing.

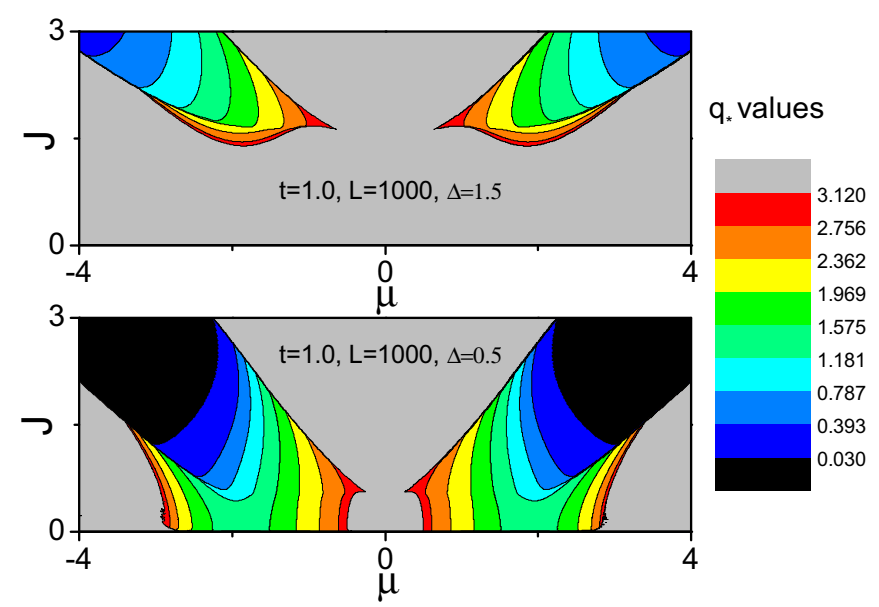

FIG. 9. (Top) Phase diagram in the chemical potential $\mu$ exchange constant $J$ plane, with fixed pairing field ( $\Delta=1.5$ ). (Bottom) Phase diagram with fixed $\Delta=0.5$.

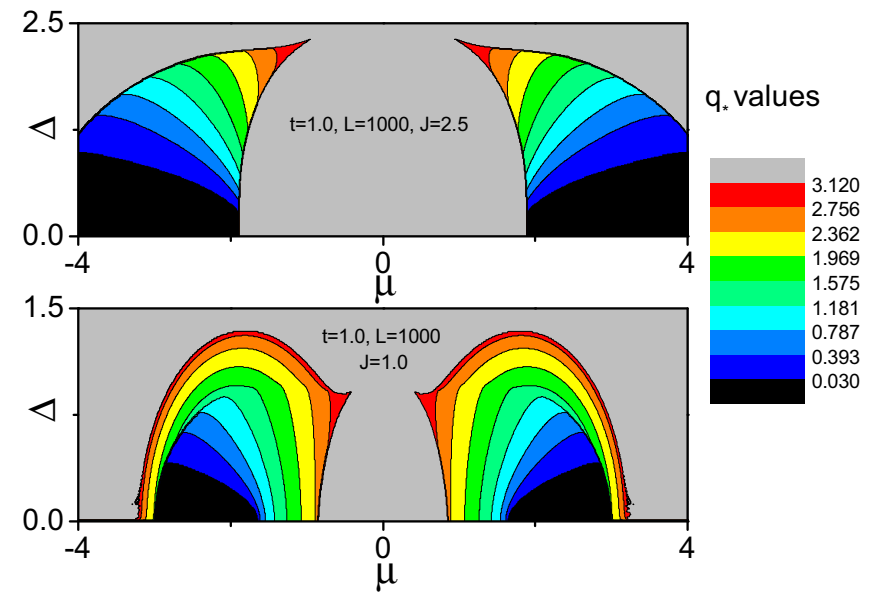

FIG. 10. (Top) Phase diagram in the chemical potential $\mu$ pairing field $\Delta$ plane, with fixed exchange constant $(J=2.5)$. (Bottom) Phase diagram with fixed $J=1.0$.

[1] S. Yunoki, J. Hu, A. L. Malvezzi, A. Moreo, N. Furukawa, and E. Dagotto, Phys. Rev. Lett. 80, 845 (1998). 